\title{
Exploring the Relationship Between Physical Activity Knowledge, Health Outcomes Expectancies, and Behavior
}

\author{
Katie M. Heinrich, Jay Maddock, and Adrian Bauman
}

\begin{abstract}
Background: Despite clear health benefits of physical activity, previous research has been limited in linking knowledge of physical activity recommendations to actual behavior. Methods: Using Expectancy Theory, we examined whether an individual's health outcome expectancies from physical activity might provide the missing link between knowledge and behavior. With data from a cross-sectional survey, we assessed differences between how much moderate physical activity people thought they needed for health benefits compared with what they thought experts recommended and the relationship of these differences to physical activity behaviors. Results: Our hypothesis that people with positive health outcome expectancies would report more minutes of physical activity than those with neutral or negative health outcome expectancies was supported for all self-reported physical activity behaviors $(P<.001)$. Conclusions: It appears that the health outcome expectancy of needing more physical activity than recommended by experts is correlated with achieving more physical activity, regardless of type. Future research should address health outcome expectancies as a way to impact physical activity.
\end{abstract}

Keywords: walking, survey, adults, moderate, vigorous

Daily physical activity is strongly related to improved health, including a reduction in all cause mortality. ${ }^{1}$ Over time the amounts of daily physical activity recommended for good health have changed. The first United States (US) health recommendations for moderate physical activity were released in 1995 advising a minimum of 30 minutes of moderate leisure time physical activity on most days of the week. ${ }^{2,3}$ In 2002, the US Institute of Medicine (IOM) released their recommendation for weight control of 60 minutes of daily moderate physical activity, with the assertion that this included all daily activities other than sleeping and breathing. ${ }^{4}$ Concerned experts from the American College of Sports Medicine (ACSM) felt that the public might be confused by the differing time requirements or may be discouraged by the 60 minute recommendation. ${ }^{5}$ In 2007, the US guidelines were clarified to indicate that 30 minutes of moderate activity on 5 days each week (or 20 minutes of vigorous activity on 3 days each week) were optimal, and bouts of at least 10 minutes could be additive to meet these guidelines. ${ }^{6}$ To attain the necessary level of physical activity, the Centers for Disease Control and Prevention (CDC) suggested the moderate and popular activity of brisk walking. ${ }^{7}$

Heinrich is with the Dept of Kinesiology, Kansas State University, Manhattan, KS. Maddock is with the Dept of Public Health Sciences, University of Hawaii, Honolulu, HI. Bauman is with the School of Public Health, Center for Physical Activity and Health, Sydney University, Sydney, Australia.
Health behavior guidelines, such as physical activity recommendations, are developed with the implicit theory that the release of the guidelines influences knowledge, which in turn affects behavior. ${ }^{8}$ Various strategies have been used to inform and educate the general public about recommendations for physical activity. The US Surgeon General took the 1995 physical activity guidelines and designed a series of public health messages ${ }^{3}$ that were further publicized by scientists and organizations. ${ }^{9}$ This type of approach was evaluated in England by asking how many days per week and minutes per day of physical activity an adult should do. The English Department of Health conducted a 3-year mass media "Active for Life" promotional campaign around their physical activity guidelines. ${ }^{10}$ This effort resulted in only a small (3\% absolute) increase in the proportion of adults who knew the correct physical activity recommendations. ${ }^{11}$

Many studies only measure if adults meet recommendations, rather than assessing their knowledge of current recommendations. ${ }^{1,12}$ In a previous US study that assessed knowledge, Morrow and associates found that although $70.4 \%$ of continental US adults knew the minimum days per week and $47.1 \%$ knew the minimum minutes per day of recommended physical activity, this knowledge was not significantly related to actual physical activity behavior. ${ }^{13}$ Canadian studies have found that only 4-5\% of adults correctly recalled physical activity recommendations, although $27-37 \%$ correctly recalled them when prompted. 8,14 Even among health and exercise professionals, recall and use of physical activity guidelines has previously found to be questionable. ${ }^{15}$ 
It appears that although some Americans may have correct knowledge of physical activity guidelines, this does not necessarily translate into physically active behaviors. Guidelines do not always explain why or how to attain the recommended amount of physical activity, ${ }^{16}$ and have used different terminology for the same actions (eg, physical activity, exercise, calisthenics, endurance). ${ }^{3}$ In fact, for adults at risk for overweight or obesity, at least 45-60 daily minutes of moderate physical activity are needed, and to prevent weight gain or regain, 60-90 daily minutes of at least moderate intensity physical activity are necessary ${ }^{17}$ Regardless of the amount of physical activity recommended by experts, it is unclear how much physical activity US adults think they personally need. What is clear is that adults who prefer and enjoy physical activity report higher physical activity levels. ${ }^{17}$ What would be the impact if individuals believe they need more physical activity for health benefits than experts recommend?

Expectancy theory highlights the relationship between cognitive outcome expectancies and behavior. ${ }^{19}$ Behaviors resulting from unobservable cognitive processes are assessed to understand learning. ${ }^{20}$ Individuals' expectations for outcomes from specific behaviors help explain the behaviors, where individuals tend to complete behaviors in ways that deliver the expected effects. It does not matter whether the outcome expectations are valid, what is more important is that they exist. ${ }^{20}$

Although knowledge of recommendations enables the behavioral capacity to perform a desired behavior, ${ }^{21}$ an individual's outcome expectancies may have a direct effect on his or her personal beliefs and behaviors. ${ }^{19}$ Thus, although inactive adults usually have similar beliefs about the benefits of physical activity as those who are sufficiently active, ${ }^{22}$ their outcome expectancies may differ. Previous research has found that increasing increments in outcome expectancies increased behavioral intention. ${ }^{23}$ Overall, outcome expectancies and physical activity behavior should be positively correlated. ${ }^{20}$

Positive outcome expectancies for health promotion can be particularly beneficial as clear relationships exist between physical activity and improved health Improving health is one reason for exercising ${ }^{24}$ and helps motivate individuals' walking and physical activity behaviors. ${ }^{25}$ Similarly, outcome expectancies of health benefits relate to exercise behavior for older adults. ${ }^{26}$ Thus, research focusing on the outcome expectancy of health benefits should predict physical activity behavior better than those focusing only on the perceived likelihood of a behavior; however, this research is lacking. ${ }^{27}$

Various studies have reported population levels of physical activity, but no one has previously examined the relationship between knowledge of physical activity recommendations, health outcome expectancies of physical activity, and physical activity behavior. Although the ACSM and CDC recommendations promote a specific amount of physical activity, ${ }^{6}$ an individual's perceived optimal dose for health benefits may differ and this outcome expectancy may correlate with actual behavior. In this study we examined the perceived amount of physical activity associated with the outcome expectancy of health benefits. We explored 2 related research questions: How much moderate physical activity do people think they need for health benefits as compared with what they think experts recommend? How does this impact their physical activity behaviors? We hypothesized that people who thought they needed more moderate physical activity for health benefits than was recommended by experts would report higher physical activity levels than those who thought they needed the same amount or less than experts' recommendations.

\section{Methods}

\section{Participants}

For this study, we used data from the 2007 Healthy Hawaii Initiative (HHI) cross-sectional random-digitdial telephone survey. All participants indicated verbal informed consent and all study procedures were approved by the university's institutional review board. Participants were 3607 adults across the state of Hawaii. Fifty-nine percent were married, $54.7 \%$ reported some college or graduating college, average age was 53.9 years, and $65.3 \%$ were female. Just over $36 \%$ of the participants were White, $28.5 \%$ were Asian, $17 \%$ were Native Hawaiian/part-Hawaiian, and $18.0 \%$ were of mixed ethnicity. Just over $4 \%$ of the sample had annual household incomes of less than $\$ 10,000,5.2 \%$ reported $\$ 10,000-19,999$, $16.7 \%$ reported $\$ 20,000-39,999,18.4 \%$ reported $\$ 40,000-59,999,36.9 \%$ reported $\$ 60,000$ or more, and $18.4 \%$ did not provide income information. The overall survey response rate was $30.3 \%$ (with $92.9 \%$ of those screened completing a survey), similar to other population level random digit dial surveys.

\section{Survey}

The development, focus, and content of the annual HHI survey have previously been described. ${ }^{28}$ Using random digit dialing procedures, the adult with the most recent birthday was asked to complete the survey, which took approximately 30 minutes. Surveys were conducted using a computer-aided telephone interview (CATI) system that controlled for skip patterns and response ranges. Survey questions covered a variety of health-related variables as well as demographics. For this study, we used a subset of the survey questions that focused on physical activity.

Rather than a 2-question item like the English Department of Health survey, ${ }^{11}$ physical activity knowledge was assessed by a single question: According to health experts, what is the minimum amount of moderate intensity activity needed per day to gain health benefits?

To assess the health outcome expectancy for physical activity, a single item was developed for this study: "For your own personal situation, what do you think is a sufficient amount of moderate-intensity physical activity needed per day to gain health benefits?" 
Using reliable and validated questions from Active Australia $^{29}$ physical activity levels were assessed. Two questions were asked to determine average minutes walked per day: "Over the last week, on how many days per week do you walk for at least 10 minutes at a time? On days when you walk for at least 10 minutes, how much total time per day do you usually spend walking?" Three questions were asked to ascertain average daily moderate physical activity: "In a usual week, do you ever do moderate activities for at least 10 minutes? How many days per week do you do these moderate activities for at least 10 minutes? On days when you do moderate activities for at least 10 minutes, how much total time per day do you usually spend doing these activities?" In addition, 3 questions were asked about vigorous physical activity: "In a usual week, do you ever do vigorous activities for at least 10 minutes? How many days per week do you do these vigorous activities for at least 10 minutes? On days per week when you do vigorous activities for at least 10 minutes, how much time in total per day do you usually spend doing these activities?"

\section{Statistical Procedures}

To determine daily minutes of each type of physical activity and to normalize distribution, all data were truncated at 240 minutes, or 4 hours per day. ${ }^{30}$ Time spent in moderate-to-vigorous physical activity was calculated as time spent in physical activities of moderate or greater intensity. We calculated descriptive statistics, including means, standard deviations, and percentages for each variable. We used chi-square and 1-way analysis of variance (ANOVA) to assess associations between demographic categories of knowledge of physical activity recommendations, health outcome expectancies for personal physical activity, physical activity behaviors, and differences between physical activity knowledge and expectancies. To address the study hypothesis, we performed 1-way ANOVAs with 3 independent variable categories: 1) people whose outcome expectancies for health benefits exceeded what they thought experts recommended (positive outcome expectancies), 2) people whose outcome expectancies for health benefits equaled what they thought experts recommended (neutral outcome expectancies), and 3) people whose outcome expectancies for health benefits were less than what they thought experts recommended (negative outcome expectancies). The dependent variables for separate ANOVAs were average daily minutes of walking, moderate physical activity, moderate-to-vigorous physical activity and vigorous physical activity.

\section{Results}

Overall, almost half (46.4\%) of the sample (unprompted) thought that health experts recommended 30 daily minutes of physical activity and $13.5 \%$ thought they recommended 60 minutes. Comparisons by participant ethnicity, income, education, and body mass index (BMI) showed that the only significant difference was that a larger proportion of underweight participants (ie, BMI $<18.5 ; 53.3 \%$ versus $43.3 \%$ for 30 minutes) thought the experts' recommendations were more than 30 minutes (data not shown). The amount of physical activity participants felt they needed per day to gain health benefits was reported as 30 minutes for $35.1 \%$, 60 minutes for $25.6 \%$, and more than 60 minutes per day for $13.1 \%$ of the sample. We found no differences by demographic categories, as the highest proportion of participants indicated that they felt they needed more than 30 minutes of moderate physical activity per day to gain health benefits in all groups (data not shown).

Physical activity distribution was divided by 10 minute increments up to those reporting 60 minutes or more each day. Table 1 shows the percentages of participants who reported each type of physical activity. The largest number of participants reported walking, and the fewest reported vigorous physical activity.

Of the 2983 people who answered both the recommendation knowledge and health outcome expectancies for moderate physical activity questions, $25.7 \%$ ( $\mathrm{n}=$ 768) thought they needed more physical activity than was recommended by experts, $49.5 \%(n=1477)$ thought they needed the same amount as recommended, and the remaining $24.7 \%(n=738)$ thought they needed less than recommended for health benefits. These percentages were calculated based on exact values rather than the recommended 30 minutes. People who thought they needed more physical activity than recommended by experts for health benefits reported more daily minutes of walking ( $r=.17, P<.001)$, were more likely to be walking at

Table 1 Percentages of Participants Who Reported Each Type of Physical Activity by Duration

\begin{tabular}{lcccc}
\hline & \multicolumn{4}{c}{ Category of daily physical activity } \\
\cline { 2 - 5 } Activity type & $<10$ minutes & $\mathbf{1 0 - 2 9}$ minutes & $\mathbf{3 0 - 5 9}$ minutes & $\geq \mathbf{6 0}$ minutes \\
\hline Walking $(\mathrm{n}=3456)$ & $30.8 \%$ & $30.0 \%$ & $20.3 \%$ & $16.8 \%$ \\
Moderate $(\mathrm{n}=2454)$ & $14.5 \%$ & $35.6 \%$ & $20.5 \%$ & $15.4 \%$ \\
Vigorous $(\mathrm{n}=1726)$ & $12.5 \%$ & $34.5 \%$ & $19.9 \%$ & $9.3 \%$ \\
Moderate-to-vigorous $(\mathrm{n}=2732)$ & $10.7 \%$ & $28.4 \%$ & $22.8 \%$ & $26.2 \%$ \\
\hline
\end{tabular}


recommended levels or more $(r=.27, P<.001)$, and were also more likely to report daily moderate physical activity at recommended levels or more $(r=.20, P<.001)$. Although the knowledge and health outcome expectancy questions queried moderate physical activity, those who thought they needed more physical activity than recommended by experts for health benefits also reported more minutes of moderate-to-vigorous physical activity $(r=$ $.12, P<.001)$ and vigorous physical activity $(r=.09, P<$ $.001)$. Table 2 displays the average daily moderate physical activity levels according to the differences between recommendations and health outcome expectancies. Note that the full range for each variable was 0-240 minutes after truncation. For all physical activity categories, as the number of minutes to achieve health outcome expectancies increased, the reported daily minutes increased.

\section{Hypothesis Test}

We found statistically significant differences in our 1-way ANOVA results between independent variable categories for each type of physical activity; daily walking $F(2,2913)$ $=60.36, P<.001$; daily minutes of moderate physical activity, $F(2,2165)=25.84, P<.001$; daily minutes of moderate-to-vigorous physical activity, $F(2,2371)=$ $20.51, P<.001$; and daily minutes of vigorous physical activity, $F(2,1524)=7.04, P=.001$. Tukey post hoc comparisons for each analysis showed that people with positive health outcome expectancies (ie, perceived amount of necessary physical activity exceeded what they thought experts recommended) were significantly more likely to report more walking than those with neutral health outcome expectancies (ie, perceived amount of necessary physical activity equaled what they thought experts recommended) and those with negative health outcome expectancies (ie, perceived amount of necessary physical activity was less than what they thought experts recommended); $P<.001$ for walking, moderate, and moderate-to-vigorous physical activity, and $P<.01$ for vigorous physical activity.

\section{Discussion}

In this study, we examined the relationship between knowledge of moderate physical activity recommendations, health outcome expectancies for moderate physical activity, and physical activity behaviors. We found support for our hypothesis that people who thought they needed more moderate physical activity for health benefits than was recommended by experts reported more daily minutes of all self-reported physical activity behaviors than those who thought they needed the same amount or less than experts' recommendations. These differences existed irrespective of the actual number of minutes the person thought were recommended by experts. This corroborated with the study by Jones and associates ${ }^{20}$ who found that it did not matter whether outcome expectations were valid, what was more important was that they existed. Unlike previous studies, we found a clear relationship between knowledge of physical activity and behavior when outcome expectancies of physical activity necessary for health benefits were included for each person. ${ }^{13,28}$ It appears that health outcome expectancies do indeed impact the relationship between knowledge and behavior for physical activity behaviors. ${ }^{19}$

Our study also helped determine the proportion of the Hawaii population with knowledge of current recommendations for moderate physical activity. Without prompting, a larger percentage of adults in our study clearly recalled the correct physical activity recommendations than Canadian adults, although the percentage who recalled the minimum minutes per day was similar to a previous US study. ${ }^{13,14}$ It is unclear if prompting

Table 2 Average Daily Moderate Physical Activity (PA) According to the Differences Between Knowledge of Recommendations and Health Outcome Expectancies

\begin{tabular}{|c|c|c|c|c|c|c|c|c|}
\hline & \multicolumn{2}{|c|}{$\begin{array}{l}\text { Average minutes } \\
\text { walked per day }\end{array}$} & \multicolumn{2}{|c|}{$\begin{array}{l}\text { Average daily minutes } \\
\text { of moderate PA }\end{array}$} & \multicolumn{2}{|c|}{$\begin{array}{l}\text { Average daily minutes of } \\
\text { moderate-to-vigorous PA }\end{array}$} & \multicolumn{2}{|c|}{$\begin{array}{c}\text { Average daily minutes } \\
\text { of vigorous PA }\end{array}$} \\
\hline & $n$ & Mean (SD) & $n$ & Mean (SD) & $n$ & Mean (SD) & $n$ & Mean (SD) \\
\hline $\begin{array}{l}\text { Negative health outcome } \\
\text { expectancy (needed less } \\
\text { than recommended for } \\
\text { health benefits) }\end{array}$ & 721 & $28(40.8)$ & 527 & $33(42.7)$ & 575 & $50(54.7)$ & 357 & $33(43.1)$ \\
\hline $\begin{array}{l}\text { Neutral health outcome } \\
\text { expectancy (needed the } \\
\text { same as recommended for } \\
\text { health benefits) }\end{array}$ & 1445 & $30(44.9)$ & 1035 & $39(51.5)$ & 1138 & $52(59.2)$ & 691 & $35(48.8)$ \\
\hline $\begin{array}{l}\text { Positive health outcome } \\
\text { expectancy (needed more } \\
\text { than recommended for } \\
\text { health benefits) }\end{array}$ & 750 & $45(54.0)$ & 606 & $48(51.5)$ & 661 & $72(64.4)$ & 477 & 44 (49.6) \\
\hline
\end{tabular}


respondents would have resulted in even higher numbers. We found that a larger proportion of underweight participants were more likely to think experts recommendations were more than 30 minutes and most participants thought they needed more than 30 minutes of daily physical activity to gain health benefits. These differences have not been previously shown.

Another change in US physical activity recommendations has occurred since the time of this study. The most recently released physical activity guidelines indicate that at least 150 minutes of moderate physical activity per week is optimal in bouts of at least 10 minutes, with 300 minutes being classified as "highly active" for additional and more extensive health benefits. ${ }^{31}$ It is unclear how this change to focus on weekly rather than daily minutes of physical activity will impact knowledge of physical activity guidelines. We plan to ask knowledge of both daily and weekly physical activity guidelines on future HHI surveys to track changes in this knowledge over time.

Using self-reported physical activity behavior may not have produced a clear picture of actual participant behavior. ${ }^{32}$ Self-report measures have been found to both over-report and under-report objectively measured physical activity. Thus, future research could objectively assess physical activity behaviors for a subsample of participants to determine the reliability of self-report in this population.

Ultimately, it may be necessary to promote the idea that individuals can gain health benefits specifically by doing more physical activity than the minimum amount recommended by experts. This could help increase the number of people with positive outcome expectancies. However, at the same time, it would be important to help provide guidance and opportunities for achieving the physical activity. Future studies could examine the effect of an intervention to increase positive outcome expectancies.

\section{Acknowledgments}

This study was made possible by a grant from the State of Hawaii Department of Health's Healthy Hawaii Initiative.

\section{References}

1. Arrieta A, Russell LB. Effects of leisure and non-leisure physical activity on mortality in U.S. adults over two decades. Ann Epidemiol. 2008;18:889-895.

2. Pate RR, Pratt M, Blair SN, et al. Physical activity and public health: a recommendation from the Centers for Disease Control and Prevention and the American College of Sports Medicine. JAMA. 1995;273:402-407.

3. United States Department of Health and Human Services. Physical activity and health: a report of the Surgeon General. Atlanta, GA: Centers for Disease Control and Prevention; 1996.

4. Institute of Medicine. Dietary reference intakes: energy, carbohydrates, fiber, fat, fatty acids, cholesterol, protein, and amino acids. Washington, DC: The National Academies Press; 2002.
5. Dickey C. ACSM applauds recognition of physical activity in new Institute of Medicine dietary guidelines, but warns of confusion and misunderstanding about IOM specifics; 2002. Accessed on August 25, 2008 at http://www. acsm.org/Content/ContentFolders/NewsReleases/2002/ ACSM_Applauds_Recognition_of_Physical_Activity_in_ New_Institute_of_Medicine_Dietary_Guidelines_But_1. htm

6. Haskell WL, Lee I-M, Pate RR, et al. Physical activity and public health: updated recommendations for adults from the American College of Sports Medicine and the American Heart Association. Med Sci Sports Exerc. 2007;89:1423-1434.

7. Centers for Disease Control and Prevention. Physical activity for everyone. Accessed August 25, 2008 at http:// www.cdc.gov/nccdphp/dnpa/physical/everyone/recommendations/

8. Bauman A, Craig CL, Cameron C. Low levels of recall among adult Canadians of the CSEP/Health Canada physical activity guidelines. Can J Appl Physiol. 2005;30:246252.

9. Bouchard C, Blair SN, Haskell WL. Physical activity and health. Champaign, IL: Human Kinetics; 2006.

10. Department of Health. Strategy statement on physical activity. London: Author; 1996.

11. Hillsdon M, Cavill N, Nanchahal K, Diamond A, White IR. National level promotion of physical activity: results from England's active for life campaign. $J$ Epidemiol Community Health. 2001;55:755-761.

12. Heinrich KM, Jokura Y, Maddock JE. Exercise selfefficacy and social norms as psychological predictors of exercise behavior. Athl Insight-J Sport Psychol. 2008;10(2): http://www.athleticinsight.com/Vol10Iss2/ ExerciseBehavior.htm.

13. Morrow JR, Krzewinski-Malone JA, Jackson AW, Bungum TJ, FitzGerald SJ. American adults' knowledge of exercise recommendations. Res Q Exerc Sport. 2004;75:231-237.

14. Cameron C, Craig CL, Bull FC, Bauman A. Canada's physical activity guidelines: has their release had an impact? Appl Physiol Nutr Metab. 2007;32:S161-S169.

15. Bauman A, Finch C. Awareness of and attitudes to the new physical activity recommendations-perceptions of attenders of the $5^{\text {th }}$ IOC world congress on sport science. J Sci Med Sport. 2000;3:493-501.

16. Brawley LR, Latimer AE. Physical activity guides for Canadians: messaging strategies, realistic expectations for change, and evaluation. Appl Physiol Nutr Metab. 2007;32:S170-S184.

17. Saris WHM, Blair SN, van Baak MA, et al. How much physical activity is enough to prevent unhealthy weight gain? Outcome of the IASO $1^{\text {st }}$ stock conference and consensus statement. Obes Rev. 2003;4:101-114.

18. Salmon J, Owen N, Crawford D, Bauman A, Sallis JF. Physical activity and sedentary behavior: a populationbased study of barriers, enjoyment, and preference. Health Psychol. 2003;22(2):178-188.

19. Bandura A. Social foundations of thought and action. Englewood Cliffs, NJ: Prentice Hall; 1986.

20. Jones BT, Corbin W, Fromme K. A review of expectancy theory and alcohol consumption. Addict. 2001;96:57-72.

21. Frederiksen LW, Martin JE, Webster JS. Assessment of smoking behavior. J Appl Behav Anal. 1979;12:653-664.

22. Carron AV, Hausenblas HA, Estabrooks PA. The psychology of physical activity. New York: McGraw Hill; 2003. 
23. Maddux JE, Sherer M, Rogers RW. Self-efficacy expectations and outcome expectancy: their relationship and their effects on behavioral intentions. Cognit Ther Res. 1982;6:1573-2819.

24. Murray L. Sport, exercise and physical activity: public participation, barriers and attitudes. Edinburgh: Information and Analytical Services, Division, Scottish Executive Education Department, 2006. http://www.scotland.gov.uk/ Resource/Doc/932/0041468.pdf.

25. Coghill N, Cooper AR. Motivators and de-motivators for adherence to a program of supported physical activity: the effect of withdrawal of professional support on sustained physical activity in middle-aged men. Prev Med. 2009; XXX: .

26. Resnick B, Palmer MH, Jenkins LS, Spellbring AM. Path analysis of efficacy expectations and exercise behaviour in older adults. J Adv Nurs. 2000;31:1309-1315.

27. Williams DM, Anderson ES, Winett RA. A review of the outcome expectancy construct in physical activity research. Ann Behav Med. 2005;29:70-79.
28. Maddock JE, Marshall CS, Nigg CR, Barnett JD. Development and first year results of a psychosocial surveillance system for chronic disease related health behaviors. Californian J Health Promot. 2003;1:54-64.

29. Brown WJ, Trost SG, Bauman A, Mummery K, Owen N. Test-retest reliability of four physical activity measures used in population surveys. J Sci Med Sport. 2004;7:205215 .

30. International Physical Activity Questionnaire. Guidelines for data processing and analysis of the international physical activity questionnaire (IPAQ) short form. Author, 2004.

31. United States Department of Health and Human Services. 2008 physical activity guidelines for Americans. ODPHP Publication No. U0036. Atlanta, GA: Centers for Disease Control and Prevention; 2008.

32. Prince SA, Adame KE, Hamel ME, Hardt J, Gorber SC, Tremblay M. A comparison of direct versus self-report measures for assessing physical activity in adults: a systematic review. Int J Behav Nutr Phys Act. 2008;5:56 http://www.ijbnpa.org/content/5/1/56. 